\title{
Nurse navigators' telemonitoring for cancer patients with COVID-19: a French case study
}

\author{
Marie Ferrua $^{1}$ (D) Delphine Mathivon ${ }^{2}$ - Adeline Duflot-Boukobza ${ }^{2} \cdot$ May Abbas $^{3} \cdot$ Cécile Charles $^{3,4}$.

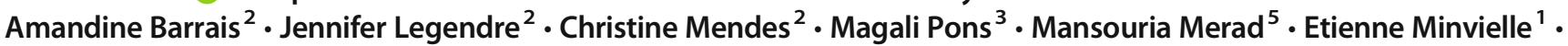 \\ Olivier Mir $^{2} \cdot$ Florian Scotté ${ }^{3}$
}

Received: 11 September 2020 / Accepted: 22 December 2020 / Published online: 18 January 2021

(C) The Author(s), under exclusive licence to Springer-Verlag GmbH, DE part of Springer Nature 2021

\begin{abstract}
Purpose The Gustave Roussy Cancer Institute implemented a patient-reported outcome platform (CAPRI-COVID) for cancer patients with coronavirus disease 2019 (COVID-19) to quarantine patients at home while ensuring monitoring of COVID-related symptoms and securing the care pathway. In this study, we described the CAPRI-COVID intervention, evaluated its use, and presented results of the tracking indicators with a focus on the nurse navigators' (NNs) activities and the experience of patients. Methods Data of 130 cancer patients with COVID-19 diagnosed from March 23 to June 5, 2020, were collected. Six COVIDrelated symptoms were monitored daily, either by the patient via the CAPRI mobile application (CAPRI App) or by NNs via telemonitoring. In the cases of worsening or new-onset symptoms, an automated alert was sent to the platform, and NNs could immediately consult an emergency physician for future course of action.

Results All 130 patients (median age: 59 years; $59.2 \%$ female) were monitored during the study period. There were no deaths or admissions to the intensive care unit attributable to COVID-19; $7.8 \%$ of patients were hospitalized (excluding scheduled hospitalization), and $17.1 \%$ were admitted to the emergency department at least once during the monitoring period. NNs carried out 1412 regular monitoring calls (average of 10.9 calls per patient), while $55 \%$ of the patients downloaded the CAPRI App. Conclusions Most patients monitored with CAPRI-COVID were quarantined during the first wave of the pandemic. In addition to the CAPRI App, which helped limit phone calls, NNs played an essential role in patient management.
\end{abstract}

Keywords COVID- $19 \cdot$ Cancer $\cdot$ Telehealth $\cdot$ Nurse navigators $\cdot$ Remote monitoring

\section{Background}

The ongoing coronavirus disease 19 (COVID-19) pandemic has created a major challenge for healthcare systems worldwide, especially in the field of oncology. In fact, cancer

Marie Ferrua

marie.ferrua@gustaveroussy.fr

1 Research Department, Gustave Roussy, Villejuif, France

2 Department of Ambulatory Cancer Care, Gustave Roussy, Villejuif, France

3 Interdisciplinary Cancer Pathways Division (DIOPP), Gustave Roussy, Villejuif, France

4 Laboratory of Psychopathology and Health Processes (EA 4057), University of Paris Descartes, Sorbonne, Paris, France

5 Emergency Care Unit, Gustave Roussy, Villejuif, France patients are considered at a higher risk of complications from COVID-19 than individuals without cancer, and the pandemic has challenged the organization of their healthcare in terms of treatment, imaging, and surgery delays [1-5].

As a counter measure, the teams of Gustave Roussy (GR), a tertiary cancer center located in Paris, France, rapidly reorganized cancer care pathways with two main objectives: (1) to protect cancer patients who may experience a severe form of COVID-19 and (ii) to ensure that these patients would continue to receive optimal cancer care [6].

Telehealth can help maintain continuity of care while limiting the exposure of patients and healthcare workers to the disease. For patients with COVID-19, digital solutions can provide remote patient monitoring to report a worsening clinical status and to offer global support to patients [7]. For an effective implementation of a telehealth solution, it is important to acknowledge the use of internet technologies in conjunction with human practices in a specific context [8]. 
Moreover, the data feedback loop is a critical component to ensure that telehealth interventions have a high impact on patient care [9]. Nurses are usually at the frontline for monitoring and providing feedback related to the remotely captured data from patients. During the COVID-19 pandemic, nurse navigators (NNs) played a major role in providing optimum healthcare to cancer patients [10-12].

In this context, GR has implemented a patient-reported outcome platform (CAPRI-COVID) for improving remote monitoring and ensuring effective management of cancer patients with COVID-19, according to the protocol defined by the French Ministry of Health [13].

CAPRI-COVID consists of a monitoring mobile application (CAPRI App) and a telephone platform with a dedicated contact number. The entire process is managed by four GR NNs. This platform has been adapted from a remote patient monitoring system device dedicated to cancer patients treated with oral anticancer therapies [8] and has recently demonstrated its clinical and medico-economic effectiveness in a randomized phase 3 study [14]. CAPRI-COVID was implemented on March 24, 2020 [15].

The first objective of this paper was to provide a comprehensive description of the CAPRI-COVID intervention; the second objective was to provide feedback on the usefulness of this platform for cancer patients by thoroughly assessing the tracking indicators, analyzing NNs activities, and assessing the experience of patients.

\section{Methods}

\section{Design and implementation of the CAPRI-COVID intervention}

The CAPRI-COVID intervention was implemented within the Coordinating Outpatient Care (Coordination des Soins Externes, CSE) unit of the Interdisciplinary Cancer Pathways Division. The CSE consists of nine NNs and one assistant nurse in contact with patients and primary care providers via a telephone platform. All NNs had French nursing grade, relevant skills in oncology, and knowledge of homecare services and healthcare system, with a dedicated post-graduation diploma. The main objectives of this unit was to organize hospital discharge at the request of inpatient department nurses (coordinating all primary care needed: private nurses, GPs, and pharmacists) and to ensure homecare monitoring via the telephone platform. Two NNs of the CSE unit were dedicated to the CAPRI telemedicine program to monitor cancer patients undergoing oral chemotherapy. This program combined a nursing-led follow-up and a mobile application (CAPRI App) for patients. NNs also provided regular (weekly for 1 month, then every other week for 6 months) telephonic follow-ups to manage symptoms and assess toxicities, adherence, and supportive care needs. All patients had access to the CAPRI App to record tracking data, contact NNs via secure messaging, or a dedicated phone line.

Faced with the COVID-19 crisis, the CAPRI-COVID intervention was implemented using tools employed for the CAPRI telemedicine program. A working group comprising physicians and NNs was initiated during the design of the intervention, and team meetings were held on a weekly basis during the project period. NNs from the CSE unit were assigned to provide remote patient monitoring. Training was organized according to standardized practices to ensure continuity of care among the NNs. The CAPRI App was upgraded to track COVID-19-related symptoms. Implementation was carried out jointly by the medical and interdisciplinary care team from the Interdisciplinary Cancer Pathways Division. A follow-up protocol was provided based on current COVID-19 guidance [13]. The protocol includes decision support tools to guide nurses in decision-making and patient orientation [15].

\section{CAPRI-COVID intervention}

The objective of the CAPRI-COVID intervention was to keep patients with COVID-19 at home as much as possible while remotely monitoring the daily evolution of COVID-related symptoms to limit irrelevant visits to the hospital and anticipate hospital visits when necessary. Furthermore, NNs supervised the discharge of patients from an inpatient unit to their homes with primary care providers to promote continuity of care. Symptom monitoring was conducted in patients via telephonic interaction (with NNs) or via the CAPRI mobile application.

\section{Patient enrolment}

Access to CAPRI-COVID was available for all patients with COVID-19. These patients were enrolled from multiple settings: following an outpatient testing for symptomatic patients, a systematic test for patients undergoing surgery, or hospitalization for COVID-19.

To avoid hospital attendance, patient consent was obtained at the time of screening, regardless of the test result. Patients also received an information leaflet presenting the intervention, contact schedules of NNs, and instructions for downloading the CAPRI mobile application.

\section{NNs activities}

NNs conducted an initial telephonic assessment interview with each patient, which included collection of clinical data related to COVID-19 and cancer and screening for psychosocial vulnerabilities. NNs were aware of the quarantine conditions for patients and verified emergency contact information in case of non-response from the patient. They trained 
patients and their relatives on the barrier measures to prevent exposure to COVID-19. They also identified the need for home assistance (meals or home care), particularly for isolated patients, and assisted them in their implementation where necessary.

NNs explained the functioning of the CAPRI-COVID intervention and the CAPRI App and sent the access login to patients. Following this interview, the NNs informed the oncologist and the GP of patients of the inclusion of the patient in the CAPRI-COVID intervention.

Patients were monitored according to the protocol defined by the French Ministry of Health, released publicly in March 2020 [13, 15]. Remote monitoring of six COVID-19related symptoms (temperature, cough, breathing discomfort, drowsiness, digestive disorders, and any new symptoms since last evaluation) was conducted and data was collected daily, either by the patient via the CAPRI App or by NNs via telemonitoring. Each contact was traced on a track sheet; for each symptom, the presence/absence and severity are reported. In cases of worsening or emergence of symptoms, an automated alert was sent to the platform; NNs then assessed the clinical condition and consulted an emergency physician when necessary. The monitoring duration was set at a minimum of 14 days (including at least 2 days with complete regression of symptoms). Monitoring was stop earlier if the patient was admitted to the hospital with worsening symptoms or scheduled hospitalization.

Patients were allowed to contact the NNs every day from 8:30 AM to 6:00 PM. They were thoroughly instructed on how to proceed in the event of worsening of symptoms, particularly in the cases of increased breathing discomfort. Additionally, they were advised to contact the emergency department of the hospital after the normal business hours of the platform.

\section{CAPRI App}

The CAPRI App is available in web or mobile versions and provides an interface to connect patients with NNs. It provides NNs with a panel to monitor the individual electronic medical records of patients. Each time a patient is contacted, NNs create intervention reports to record their activities and observations. Each intervention performed by the NNs is subsequently tracked on the platform.

\section{Data collection}

Data were collected from March 23 to June 5, 2020. Data concerning clinical symptoms, hospitalizations, and emergency visits were retrieved from the medical records. Interventions performed by NNs and patient login were extracted from the CAPRI App.
The experience of patients was measured by a survey administered via secured e-mail or by post for those without a valid email address in June 2020. The survey included 25 items on 4 themes: expectations of CAPRI-COVID, benefits of CAPRI-COVID, behavior of NNs, and the CAPRI App.

\section{Data analysis}

A coding grid was developed to extract the information identified during monitoring from the NNs intervention reports and to categorize the actions subsequently implemented. NNs' interventions were independently coded by nurses and researchers.

Data were recorded on a spreadsheet (Microsoft Excel®), and a database was incorporated. The data were then summarized using descriptive statistics.

The study was conducted with approval of the Institutional Review Board (CSET), in agreement with applicable laws and regulations and the Helsinki Declaration.

\section{Tracking indicators}

Indicators were defined to follow the evolution of symptoms in patients with COVID-19. The primary indicator was the proportion of patients who were admitted to the hospital. Other indicators included the number of emergency visits, hospitalizations in the intensive care unit, and deaths related to COVID-19.

\section{Results}

\section{Population}

As a part of the CAPRI-COVID intervention, 130 patients with COVID-19 were monitored; findings from 129 are presented in Table 1 (one patient requested the withdrawal of their consent for the use of personal data). Sixty-two patients completed the survey, with a response rate of $48.1 \%$. Fiftyfive percent of patients were enrolled after hospitalization for COVID-19 and $45.0 \%$ after RT-PCR test performed in the outpatient department (Fig. 1). Among all patients, 19.4\% did not show symptoms of COVID-19. The median time from symptom onset to the start of monitoring was 12 (1-66) days, with a mean duration of 14.7 (5-39) days.

\section{Tracking indicators}

There were no deaths or admissions to the intensive care unit attributable to COVID-19. Ten (7.8\%) patients were hospitalized (excluding scheduled hospitalization), and 23 (17.1\%) were admitted to the emergency department at least once 
Table 1 Baseline characteristics of patients

\begin{tabular}{|c|c|c|c|}
\hline Variables & Phone use $(n=79)$ & CAPRI App use $(n=50)$ & Total patients $(n=129)$ \\
\hline Age, mean (SD), years & $60(14)$ & $55(16)$ & $59(15)$ \\
\hline Women, $n(\%)$ & $47(59.5)$ & $29(58.0)$ & $76(58.9)$ \\
\hline BMI $\left(\mathrm{kg} / \mathrm{m}^{2}\right)$, mean (range) & $25.5(15.8-46.4)$ & $23.8(14.9-34.0)$ & $24.9(14.9-46.4)$ \\
\hline \multicolumn{4}{|l|}{ Tumor site $n(\%)$} \\
\hline Breast & $17(21.5)$ & $10(20.0)$ & $27(20.9)$ \\
\hline Head and neck & $15(19.0)$ & $5(10.0)$ & $20(15.5)$ \\
\hline Hematological & $8(10.1)$ & $8(16.0)$ & $16(12.4)$ \\
\hline Gynecological & $10(12.7)$ & $4(8.0)$ & $14(10.9)$ \\
\hline Digestive & $7(8.9)$ & $6(12.0)$ & $13(10.1)$ \\
\hline Renal & $5(6.3)$ & $4(8.0)$ & $9(7.0)$ \\
\hline Skin & $3(3.8)$ & $5(10.0)$ & $8(6.2)$ \\
\hline Lung & $4(5.1)$ & $3(6.0)$ & $7(5.4)$ \\
\hline Other & $7(8.9)$ & $0(0.0)$ & $7(5.4)$ \\
\hline Non-cancer patient & $3(3.8)$ & $5(10.0)$ & $8(6.2)$ \\
\hline \multicolumn{4}{|l|}{ Current treatment $n(\%)$} \\
\hline Yes & $36(45.6)$ & $25(50.0)$ & $61(46.8)$ \\
\hline Chemotherapy & $15(11.6)$ & $16(12.4)$ & $31(24.0)$ \\
\hline Immunotherapy & $5(3.9)$ & $3(2.3)$ & $8(6.2)$ \\
\hline Hormonal therapy & $5(3.9)$ & $5(3.9)$ & $10(7.8)$ \\
\hline Oral anticancer agents & $6(4.7)$ & $0(0.0)$ & $6(4.7)$ \\
\hline Radiotherapy & $3(2.3)$ & $0(0.0)$ & $3(2.3)$ \\
\hline Other & $2(1.6)$ & $1(0.8)$ & $3(2.3)$ \\
\hline Pending treatment & $16(20.3)$ & $12(24.0)$ & $28(22.2)$ \\
\hline No & $27(34.2)$ & $13(26.0)$ & $40(31.0)$ \\
\hline
\end{tabular}

$B M I$ body mass index

during monitoring. Among hospitalized patients, 50\% had already been hospitalized for COVID-19.

\section{NNs activities}

NNs conducted 1412 regular telemonitoring calls to assess the evolution of COVID-19-related symptoms, with an average of 10.9 calls per patient. For patients using the CAPRI App, the number of calls decreased to 6.2 calls per patient compared to 13.9 for those monitored via phone. NNs received 35 incoming calls from 22 patients (13 patients monitored via phone and 9 via CAPRI App). Thus, 1447 interventions were analyzed.

Regarding the evolution of COVID-19-related symptoms, 53 interventions (30 patients) required medical consultation from an emergency physician. Twenty-eight patients were
Fig. 1 Patient enrolment and monitoring in CAPRI-COVID




referred to the emergency department and three to the GP (general practitioner). Prolonged symptomatic surveillance was suggested in 22 cases. During the monitoring, NNs consulted the oncologist in 37 interventions (involving 28 patients). Referrals concerning requests for cancer treatment management (questions about treatment modifications or sideeffects) occurred in $62.2 \%$ of cases.

Screening for psychosocial vulnerabilities resulted in the referral of 22 patients to the psycho-oncology unit and/or the social service of the hospital. NNs also detected significant weight loss during follow-up and referred 30 patients for dietary support. In addition, NNs intervened to implement home care in eight patients. The CAPRI-COVID intervention involved an average of two full-time NNs, 7 days a week, over a total duration of 70 days.

\section{CAPRI App}

Only $114(88.4 \%)$ patients had a valid e-mail address (required to access the CAPRI App) at inclusion. Among them, $71(62.3 \%)$ downloaded the CAPRI App, and 50 (43.9\%) completed the tracking data (average 16 times, 1.1 times per day) (Fig. 1). Three patients were not compliant with the monitoring via the CAPRI App and switched to phone monitoring.

The rate of responses to the survey indicates that patients using the CAPRI App were overall satisfied and did not consider the procedure too constraining $(n=32)$ (Fig. 2). The main reason given by patients for not downloading the CAPRI App was their lack of familiarity with computers/smartphones (cf. Table 2). Patients who used the CAPRI App infrequently preferred regular phone contact with NNs $(n=7)$.

\section{Patient experience}

Among the 62 responders to the patient survey, $61.7 \%$ were women. Nine percent of the patients were under 45 years old, $32.8 \%$ were aged $45-59,39.3 \%$ were aged $60-75$, and $13.1 \%$ were over 75 years old. Regarding the number of people in the household, $18.3 \%$ of the patients had 1, $45.0 \%$ had 2, 6.7\% had 3 , and $30.0 \%$ had more than 3 people.

Table 2 Patients' reasons for not downloading the CAPRI App

\begin{tabular}{ll}
\hline Reasons for not downloading the CAPRI App $(n=28)$ & $n(\%)$ \\
\hline I am not familiar with computers/my smartphone & $9(32.1)$ \\
I do not have a smartphone & $5(17.9)$ \\
I tried, but I could not download it & $3(10.7)$ \\
I did not want to fill out the forms every day & $3(10.7)$ \\
I wanted to talk to someone & $3(10.7)$ \\
I have not found the need for it & $2(7.1)$ \\
I did not trust the use of my data & $1(3.6)$ \\
Other reason & $2(7.1)$ \\
\hline
\end{tabular}

Patients expressed very positive interest in the CAPRICOVID intervention (Fig. 3), and $96.7 \%$ of patients reported that the frequency of calls from the NNs was adequate.

On the general impression of the CAPRI-COVID intervention (open-ended question), patients highlighted the importance of human contact and being listened to. They felt the monitoring was very reassuring in the context of the pandemic.

\section{Discussion}

Most patients monitored with CAPRI-COVID were quarantined at home during the first wave of the COVID-19 pandemic ( $92.2 \%$ of patients).

The intervention was quickly and successfully implemented first because the hospital already had a unit with NNs with the skills and experience necessary for remote cancer patient monitoring. Second, the pre-existing CAPRI App and organizational tools (such as protocol and clinical decision support) used to monitor patients receiving oral therapies were rapidly adapted by the network combining the medical and care teams $[8,14,16]$. The implementation of the intervention was unprecedented in this context of health crisis due to uncertainty about the duration of the study and the number of patients expected to be enrolled. Weekly meetings were essential to discuss the problems encountered and to adapt the intervention protocol. NNs were able to ensure remote overall patient care not only from a clinical point of view but also in the organization of the pathway as well as for education and prevention, in the context of the COVID-19 pandemic. They personalized remote monitoring according to the type of pathology, current treatment, family context, and the level of understanding of patients. These nursing skills have previously been developed in other discussions on handling crises related to COVID-19 $[12,17]$. Due to their skills, NNs have an emerging role in the implementation of telehealth solutions [18]. They were equally able to play a key role in innovation systems linked with the use of new technologies, which usually require a human central organizer for each patient [19-22].

With regard to the clinical aspect of the study, NNs provided monitoring and grading of COVID-19 symptoms to initiate three main actions: providing advice to patients, referring them to primary care, or requesting further evaluation from an emergency physician for an eventual visit or hospitalization. This enabled a practical adjustment of hospital visits in the context of a lockdown related to COVID-19.

In addition to the monitoring of symptoms related to COVID-19 planned in the protocol, NNs identified vulnerabilities that required the implementation of targeted actions, mainly of three types: (1) the link with the referring oncologist for cancer follow-up and the implementation of supportive 
Fig. 2 Patients' perception of the CAPRI App



care, (2) a screening of psycho-social vulnerabilities and orientation if necessary, and (3) the link with primary care professionals and caregivers in the home of patients.

NNs connected with the referral oncologist for cancer management, on associated treatments and investigations as well as the implementation of supportive care when necessary. Weight loss, a key point of concern in oncology, has been identified as critical for patients. After discussion with the working group, routine screening for weight loss issues was added to the initial assessment to implement dietary measures.

The context of COVID-19 pandemic has been very stressful for cancer patients who are considered "frail population" [5] and therefore are subject to the risk of stress-inducing withdrawal complications. Some treatments had to be canceled or postponed, in favor of the COVID-19 treatment. In response to this anxiety, routine screening and early referral to onco-psychology services were implemented.
NNs are also linked to primary care by providing information to GPs, private nurses, pharmacists, and home care facilities when needed, particularly for patients admitted to hospitals to organize and secure the patient's return based on health requirements. Although the intervention was implemented for patients affected by COVID-19, the role of the NNs focused on assisting patients in all dimensions of care (physical, emotional, psychosocial, and family) identified by Pautasso et al. [23]. Interventions involving NNs have already proven their effectiveness in improving quality of life and hospital satisfaction and decreased hospital stay for patients with cancer in previous studies [24-26]. In our study, the absence of a control group did not provide an assessment of the potential added value.

The value of the intervention lies in the combination of the use of the CAPRI App with the NN's telephone tracking system. Therefore, due to this application, the number of calls was reduced and due assistance could be provided to patients
Fig. 3 Patients' perceptions of the CAPRI-COVID intervention $(n=$ 62)

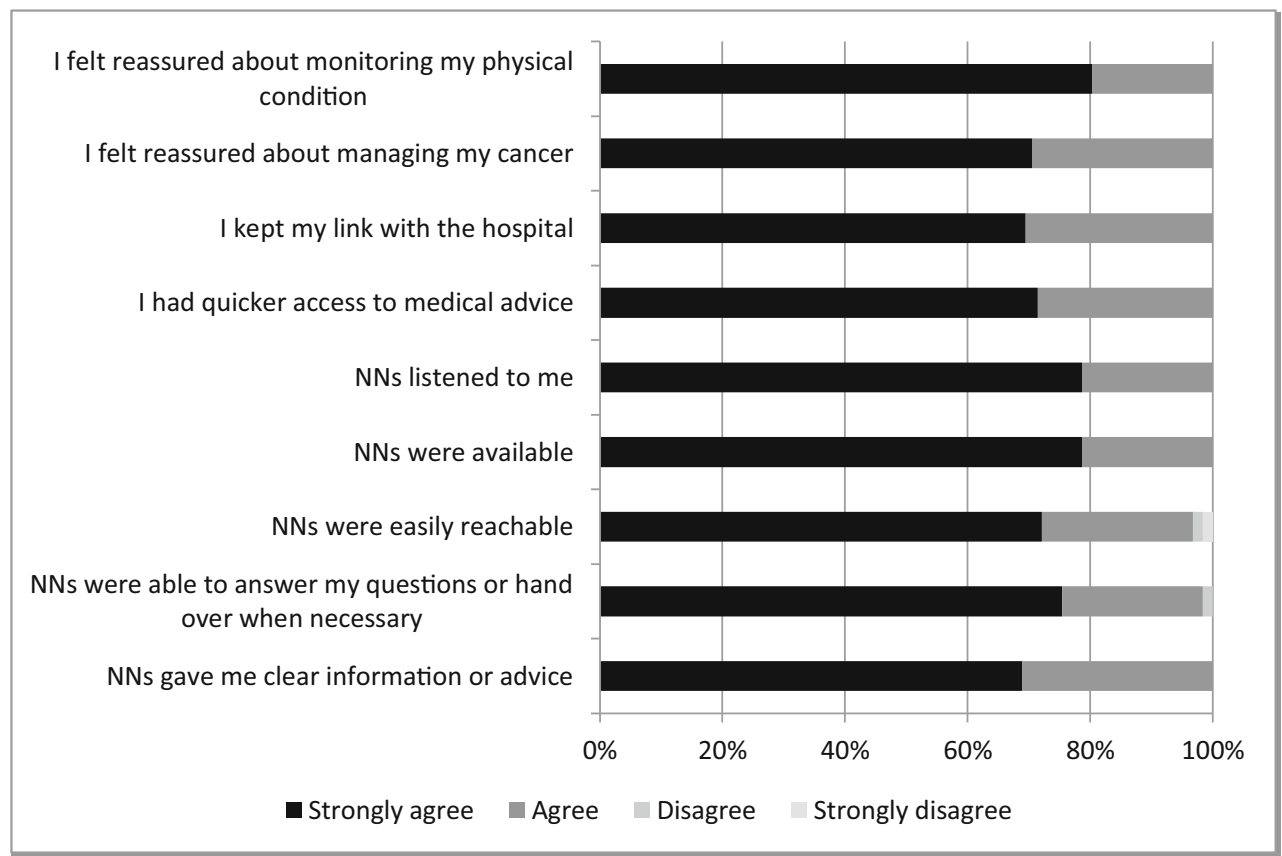


in need. However, we observed that some patients were reluctant to use the application either because they were unfamiliar with the new technology or because they preferred human contact.

Patient attitude was also similar in the CAPRI study [27] previously conducted in patients receiving oral therapies, in which we had observed at the midpoint that $53 \%$ of patients reported having attempted to connect and only $38 \%$ reported monitoring data at least once.

Although a responder rate of $48 \%$ could represent a selection bias, patients highlighted the importance of careful listening, having a contact person who is easily reachable, available, and empathetic. These patient reports were similar to those observed in the CAPRI study [27].

The main limitation of the CAPRI-COVID intervention lies in the fact that phone calls are time-consuming and require dedicated nursing time. In addition, it was necessary to ensure telephonic support and monitoring on weekends. Moreover, phone or app monitoring does not always allow an accurate evaluation of certain clinical situations that could be facilitated by a video contact.

\section{Conclusion and perspectives}

The majority of patients monitored with CAPRI-COVID were maintained at home during the first wave of the COVID-19 pandemic. The monitoring program helped to ensure safe cancer care pathways during this period by avoiding unnecessary visits to the hospital while ensuring the monitoring of symptoms. NNs played an essential role in addition to the use of the CAPRI App. They provided personalized care to the patients by managing the course of COVID-19 symptoms and contributed to a global approach in patient management. NNs were able to identify vulnerabilities and implement preventive measures. The use of the CAPRI App by a group of patients permitted a reduction in the number of phone calls.

Having a pre-existing organization also facilitated the implementation of a patient-reported outcome platform. Involving NNs with skills to ensure the remote monitoring of cancer patients at home was particularly useful. Other adaptations of the CAPRI intervention might be expanded in the future to extend to other types of patient support programs.

Acknowledgments The authors acknowledge the Fondation de France for its financial support and the Medialis Company for the development of the CAPRI App. The authors are most grateful to Richard Medeiros, Medical Editing International, for editing the manuscript. The authors would also like to thank Dr. Sarah Dauchy for her support.

Authors' contributions All authors contributed to the platform development. Material preparation, data collection, and analysis were performed by MF, DM, AD, JL, CM, and AB. The first draft of the manuscript was written by MF, and all authors commented on previous versions of the manuscript. OM, EM, and FS made a substantial contribution to preparation of the manuscript. All authors read and approved the final manuscript.

Funding Part of this research was supported by the Fondation de France.

Data availability The data sets analyzed in this study are available from the corresponding author on request.

\section{Compliance with ethical standards}

Conflict of interest OM has received consultancy fees from Amgen, AstraZeneca, Bayer, Blueprint Medicines, Bristol-Myers Squibb, Eli Lilly, Ipsen, Lundbeck, Merck Sharpe \& Dohme, Pfizer, Roche, Servier, and Vifor Pharma. He is a shareholder of Amplitude Surgical, Ipsen, and Transgene. F.S. has a conflict of interests with Mylan, BMS, Tesaro, Sanofi, Roche, MSD, TEVA, Norgine, Prostrakan, Leo Pharma, Janssen, Hospira, Boehringer, Amgen, Pierre Fabre Oncologie, Vifor Pharma, Arrow, and Mundi Pharma. Other authors have no conflict of interest to declare.

Ethics approval The study was conducted after approval of the Institutional Review Board (CSET), in agreement with applicable laws and regulations, and according to the Helsinki Declaration.

Consent to participate All patients enrolled in the study completed a written consent form prior to their participation in the study.

Consent for publication All patients enrolled in the study completed a written consent form for the use and publication of their data.

Code availability Not applicable.

\section{References}

1. Tanne JH, Hayasaki E, Zastrow M, Pulla P, Smith P, Rada AG (2020) Covid-19: how doctors and healthcare systems are tackling coronavirus worldwide. BMJ (Clin Res Ed) 368:m1090. https://doi. org/10.1136/bmj.m1090

2. Liang W, Guan W, Chen R, Wang W, Li J, Xu K, Li C, Ai Q, Lu W, Liang H, Li S, He J (2020) Cancer patients in SARS-CoV-2 infection: a nationwide analysis in China. Lancet Oncol 21:335337. https://doi.org/10.1016/S1470-2045(20)30096-6

3. Schrag D, Hershman DL, Basch E (2020) Oncology practice during the COVID-19 pandemic. JAMA 323:2005-2006. https://doi.org/ 10.1001/jama.2020.6236

4. van de Haar J, Hoes LR, Coles CE, Seamon K, Fröhling S, Jäger D, Valenza F, de Braud F, De Petris L, Bergh J, Ernberg I, Besse B, Barlesi F, Garralda E, Piris-Giménez A, Baumann M, Apolone G, Soria JC, Tabernero J, Caldas C, Voest EE (2020) Caring for patients with cancer in the COVID-19 era. Nat Med 26:665-671. https://doi.org/10.1038/s41591-020-0874-8

5. Dai M, Liu D, Liu M, Zhou F, Li G, Chen Z, Zhang Z, You H, Wu M, Zheng Q, Xiong Y, Xiong H, Wang C, Chen C, Xiong F, Zhang Y, Peng Y, Ge S, Zhen B, Yu T, Wang L, Wang H, Liu Y, Chen Y, Mei J, Gao X, Li Z, Gan L, He C, Li Z, Shi Y, Qi Y, Yang J, Tenen DG, Chai L, Mucci LA, Santillana M, Cai H (2020) Patients with cancer appear more vulnerable to SARS-CoV-2: a multicenter study during the COVID-19 outbreak. Cancer Discov 10:783791. https://doi.org/10.1158/2159-8290.CD-20-0422 
6. Barlesi F, Foulon S, Bayle A, Gachot B, Pommeret F, Willekens C, Stoclin A, Merad M, Griscellil F, Micol J, Sun R, Nihouarn T, Balleygier C, André F, Scotte F, Besse B, Soria J, Albiges L (2020) Outcome of cancer patients infected with COVID-19, including toxicity of cancer treatments. In: AACR Annual Meeting

7. Hollander JE, Carr BG (2020) Virtually perfect? Telemedicine for Covid-19. N Engl J Med 382:1679-1681. https://doi.org/10.1056/ NEJMp2003539

8. Ferrua M, Minvielle E, Fourcade A, Lalloué B, Sicotte C, Di Palma M, Mir O (2020) How to design a remote patient monitoring system? A French case study. BMC Health Serv Res 20:434. https:// doi.org/10.1186/s12913-020-05293-4

9. Vegesna A, Tran M, Angelaccio M, Arcona S (2017) Remote patient monitoring via non-invasive digital technologies: a systematic review. Telemed J E Health 23:3-17. https://doi.org/10.1089/tmj. 2016.0051

10. Nalley C (2020) Navigating the COVID-19 pandemic as an oncology nurse. Oncol Times 42:11. https://doi.org/10.1097/01.COT. 0000661864.55789.d7

11. Nuryani SNA, Lisnawati LG (2020) Nurse's roles in protecting cancer patients during COVID-19 pandemic. JBN 4:25. https:// doi.org/10.24843/JBN.2020.v04.is01.p06

12. Paterson C, Gobel B, Gosselin T, Haylock PJ, Papadopoulou C, Slusser K, Rodriguez A, Pituskin E (2020) Oncology nursing during a pandemic: critical reflections in the context of COVID-19. Semin Oncol Nurs 36:151028. https://doi.org/10.1016/J.SONCN. 2020.151028

13. Ministère des solidarités et de la santé coronavirus - Ministère des Solidarités et de la Santé. https://solidarites-sante.gouv.fr/soins-etmaladies/maladies/maladies-infectieuses/coronavirus/. Accessed Oct 162020

14. Mir O, Ferrua M, Fourcade A, Mathivon D, Duflot-Boukobza A, Dumont S, Baudin E, Delaloge S, Malka D, Albiges L, Pautier P, Robert C, Planchard D, De Botton S, Lemare F, Guillet M, Puglisi V, Abbas M, Di Palma M, Minvielle E (2020) Intervention combining nurse navigators (NNs) and a mobile application versus standard of care (SOC) in cancer patients (pts) treated with oral anticancer agents (OAA): results of CapRI, a single-center, randomized phase III trial. J Clin Oncol. https://doi.org/10.1200/JCO.2020. 38.15_suppl.2000

15. Scotté F, Minvielle E, Mir O, André F, Barlesi F, Soria JC (2020) A patient reported outcome platform, a useful tool to improve monitoring and effective management of Covid-19-positive patients with cancer. Eur J Cancer 132:1-4. https://doi.org/10.1016/J. EJCA.2020.03.020

16. Yatim F, Cristofalo P, Ferrua M, Girault A, Lacaze M, Di Palma M, Minvielle E (2017) Analysis of nurse navigators' activities for hospital discharge coordination: a mixed method study for the case of cancer patients. Support Care Cancer 25:863-868. https://doi.org/ 10.1007/s00520-016-3474-x
17. McLaughlin-Barrett S (2020) Outnumbered yet needed: the lung cancer nurse specialist. Respirology 13844. https://doi.org/10.1111/ resp. 13844

18. Emfield Rowett K, Christensen D (2020) Oncology nurse navigation: expansion of the navigator role through telehealth. Clin J Oncol Nurs 24:24-31. https://doi.org/10.1188/20.CJON.S1.24-31

19. Moser EC, Narayan G (2020) Improving breast cancer care coordination and symptom management by using AI driven predictive toolkits. Breast 50:25-29. https://doi.org/10.1016/j.breast.2019.12. 006

20. Adler G, Kaufman G, Simon-Tuval T (2019) Healthcare utilization of breast cancer patients following telephone-based consultations of oncology nurse navigator via telemedical care. PLoS One 14: e0216365. https://doi.org/10.1371/journal.pone.0216365

21. Zwingerman R, Melenchuk K, McMahon E, Liu KE, Siren A, Laferriere N, Greenblatt EM (2020) Expanding urgent oncofertility services for reproductive age women remote from a tertiary level Fertility Centre by use of telemedicine and an on-site nurse navigator. J Cancer Educ 35:515-521. https://doi.org/10.1007/s13187019-01490-w

22. Tietjen KM, Breitenstein S (2017) A nurse-led telehealth program to improve emotional health in individuals with multiple sclerosis. J Psychosoc Nurs Ment Health Serv 55:31-37. https://doi.org/10. 3928/02793695-20170301-04

23. Pautasso FF, Zelmanowicz AM, Flores CD, Caregnato RCA (2018) Role of the nurse navigator: integrative review. Rev Gaúcha Enferm 39:e20170102. https://doi.org/10.1590/1983-1447.2018. 2017-0102

24. Lee T, Ko I, Lee I, Kim E, Shin M, Roh S, Yoon D, Choi S, Chang $\mathrm{H}$ (2011) Effects of nurse navigators on health outcomes of cancer patients. Cancer Nurs 34:376-384. https://doi.org/10.1097/NCC. 0b013e3182025007

25. Yackzan S, Stanifer S, Barker S, Blair B, Glass A, Weyl H, Wheeler P (2019) Outcome measurement: patient satisfaction scores and contact with oncology nurse navigators. Clin J Oncol Nurs 23:7681. https://doi.org/10.1188/19.CJON.76-81

26. Tuominen L, Stolt M, Meretoja R, Leino-Kilpi H (2019) Effectiveness of nursing interventions among patients with cancer: an overview of systematic reviews. J Clin Nurs 28:2401-2419. https://doi.org/10.1111/jocn.14762

27. Ferrua M, di Palma M, Fourcade A, Guillet M, Mathivon D, Puglisi V, Mir O, Minvielle E (2019) Patient experience and use of an intervention combining nurse-led telephone and technologies for the monitoring of oral cancer medication. Ann Oncol 30. https:// doi.org/10.1093/annonc/mdz272.011

Publisher's note Springer Nature remains neutral with regard to jurisdictional claims in published maps and institutional affiliations. 